\title{
Weighted boundedness of a multilinear operator associated to a singular integral operator with general kernels
}

\section{Qiufen Feng*}

\section{"Correspondence:} fengqiufen@126.com Changsha Commerce and Tourism College, Changsha, 410116, P.R. China

\section{Springer}

\begin{abstract}
In this paper, we establish the weighted sharp maximal function inequalities for a multilinear operator associated to a singular integral operator with general kernels. As an application, we obtain the boundedness of the operator on weighted Lebesgue and Morrey spaces.

MSC: 42B20; 42B25

Keywords: multilinear operator; singular integral operator; sharp maximal function; weighted $B M O$; weighted Lipschitz function
\end{abstract}

\section{Introduction and preliminaries}

As the development of singular integral operators (see [1-3]), their commutators and multilinear operators have been well studied. In [4-6], the authors prove that the commutators generated by singular integral operators and $B M O$ functions are bounded on $L^{p}\left(R^{n}\right)$ for $1<p<\infty$. Chanillo (see [7]) proves a similar result when singular integral operators are replaced by fractional integral operators. In $[8,9]$, the boundedness for the commutators generated by singular integral operators and Lipschitz functions on Triebel-Lizorkin and $L^{p}\left(R^{n}\right)(1<p<\infty)$ spaces is obtained. In [10,11], the boundedness for the commutators generated by singular integral operators and weighted $B M O$ and Lipschitz functions on $L^{p}\left(R^{n}\right)(1<p<\infty)$ spaces is obtained. In [12], some singular integral operators with general kernel are introduced, and the boundedness for the operators and their commutators generated by $B M O$ and Lipschitz functions is obtained (see $[8,12,13])$. Motivated by these, in this paper, we study the multilinear operator generated by the singular integral operator with general kernel and the weighted Lipschitz and $B M O$ functions.

First, let us introduce some notations. Throughout this paper, $Q$ denotes a cube of $R^{n}$ with sides parallel to the axes. For any locally integrable function $f$, the sharp maximal function of $f$ is defined by

$$
M^{\#}(f)(x)=\sup _{Q \ni x} \frac{1}{|Q|} \int_{Q}\left|f(y)-f_{Q}\right| d y,
$$

where, and in what follows, $f_{Q}=|Q|^{-1} \int_{Q} f(x) d x$. It is well known that (see $\left.[1,2]\right)$

$$
M^{\#}(f)(x) \approx \sup _{Q \ni x} \inf _{c \in C} \frac{1}{|Q|} \int_{Q}|f(y)-c| d y .
$$

@2014 Feng; licensee Springer. This is an Open Access article distributed under the terms of the Creative Commons Attribution License (http://creativecommons.org/licenses/by/2.0), which permits unrestricted use, distribution, and reproduction in any medium, provided the original work is properly cited. 
Let

$$
M(f)(x)=\sup _{Q \ni x} \frac{1}{|Q|} \int_{Q}|f(y)| d y .
$$

For $\eta>0$, let $M_{\eta}^{\#}(f)(x)=M^{\#}\left(|f|^{\eta}\right)^{1 / \eta}(x)$ and $M_{\eta}(f)(x)=M\left(|f|^{\eta}\right)^{1 / \eta}(x)$.

For $0<\eta<n, 1 \leq p<\infty$ and the non-negative weight function $w$, set

$$
M_{\eta, p, w}(f)(x)=\sup _{Q \ni x}\left(\frac{1}{w(Q)^{1-p \eta / n}} \int_{Q}|f(y)|^{p} w(y) d y\right)^{1 / p} .
$$

We write $M_{\eta, p, w}(f)=M_{p, w}(f)$ if $\eta=0$.

The $A_{p}$ weight is defined by (see [1]), for $1<p<\infty$,

$$
A_{p}=\left\{w \in L_{\mathrm{loc}}^{1}\left(R^{n}\right): \sup _{Q}\left(\frac{1}{|Q|} \int_{Q} w(x) d x\right)\left(\frac{1}{|Q|} \int_{Q} w(x)^{-1 /(p-1)} d x\right)^{p-1}<\infty\right\}
$$

and

$$
A_{1}=\left\{w \in L_{\mathrm{loc}}^{p}\left(R^{n}\right): M(w)(x) \leq C w(x), \text { a.e. }\right\} .
$$

Given a non-negative weight function $w$, for $1 \leq p<\infty$, the weighted Lebesgue space $L^{p}\left(R^{n}, w\right)$ is the space of functions $f$ such that

$$
\|f\|_{L^{p}(w)}=\left(\int_{R^{n}}|f(x)|^{p} w(x) d x\right)^{1 / p}<\infty .
$$

For $0<\beta<1$ and the non-negative weight function $w$, the weighted Lipschitz space $\operatorname{Lip}_{\beta}(w)$ is the space of functions $b$ such that

$$
\|b\|_{L_{i p_{\beta}(w)}}=\sup _{Q} \frac{1}{w(Q)^{\beta / n}}\left(\frac{1}{w(Q)} \int_{Q}\left|b(y)-b_{Q}\right|^{p} w(x)^{1-p} d y\right)^{1 / p}<\infty,
$$

and the weighted $B M O$ space $B M O(w)$ is the space of functions $b$ such that

$$
\|b\|_{B M O(w)}=\sup _{Q}\left(\frac{1}{w(Q)} \int_{Q}\left|b(y)-b_{Q}\right|^{p} w(x)^{1-p} d y\right)^{1 / p}<\infty .
$$

Remark (1) It has been known that (see [11, 14]), for $b \in \operatorname{Lip}_{\beta}(w), w \in A_{1}$ and $x \in Q$,

$$
\left|b_{Q}-b_{2^{k} Q}\right| \leq C k\|b\|_{L i p_{\beta}(w)} w(x) w\left(2^{k} Q\right)^{\beta / n} .
$$

(2) It has been known that (see [2, 14]), for $b \in B M O(w), w \in A_{1}$ and $x \in Q$,

$$
\left|b_{Q}-b_{2^{k} Q}\right| \leq C k\|b\|_{B M O(w)} w(x) .
$$

(3) Let $b \in \operatorname{Lip}_{\beta}(w)$ or $b \in B M O(w)$ and $w \in A_{1}$. By [14], we know that spaces $\operatorname{Lip}_{\beta}(w)$ or $B M O(w)$ coincide and the norms $\|b\|_{L i p_{\beta}(w)}$ or $\|b\|_{B M O(w)}$ are equivalent with respect to different values $1 \leq p<\infty$. 
Definition 1 Let $\varphi$ be a positive, increasing function on $R^{+}$, and there exists a constant $D>0$ such that

$$
\varphi(2 t) \leq D \varphi(t) \quad \text { for } t \geq 0
$$

Let $w$ be a non-negative weight function on $R^{n}$ and $f$ be a locally integrable function on $R^{n}$. Set, for $1 \leq p<\infty$,

$$
\|f\|_{L^{p, \varphi}(w)}=\sup _{x \in R^{n}, d>0}\left(\frac{1}{\varphi(d)} \int_{Q(x, d)}|f(y)|^{p} w(y) d y\right)^{1 / p},
$$

where $Q(x, d)=\left\{y \in R^{n}:|x-y|<d\right\}$. The generalized weighted Morrey space is defined by

$$
L^{p, \varphi}\left(R^{n}, w\right)=\left\{f \in L_{\mathrm{loc}}^{1}\left(R^{n}\right):\|f\|_{L^{p, \varphi}(w)}<\infty\right\} .
$$

If $\varphi(d)=d^{\delta}, \delta>0$, then $L^{p, \varphi}\left(R^{n}, w\right)=L^{p, \delta}\left(R^{n}, w\right)$, which is the classical Morrey space (see $[15,16])$. If $\varphi(d)=1$, then $L^{p, \varphi}\left(R^{n}, w\right)=L^{p}\left(R^{n}, w\right)$, which is the weighted Lebesgue space (see [1]).

As the Morrey space may be considered as an extension of the Lebesgue space, it is natural and important to study the boundedness of the operator on the Morrey spaces (see [17-20]).

In this paper, we study some singular integral operators as follows (see [12]).

Definition 2 Let $T: S \rightarrow S^{\prime}$ be a linear operator such that $T$ is bounded on $L^{2}\left(R^{n}\right)$ and has a kernel $K$, that is, there exists a locally integrable function $K(x, y)$ on $R^{n} \times R^{n} \backslash\{(x, y) \in$ $R^{n} \times R^{n}: x=y$ such that

$$
T(f)(x)=\int_{R^{n}} K(x, y) f(y) d y
$$

for every bounded and compactly supported function $f$, where $K$ satisfies

$$
\begin{aligned}
& |K(x, y)| \leq C|x-y|^{-n}, \\
& \int_{2|y-z|<|x-y|}(|K(x, y)-K(x, z)|+|K(y, x)-K(z, x)|) d x \leq C,
\end{aligned}
$$

and there is a sequence of positive constant numbers $\left\{C_{k}\right\}$ such that for any $k \geq 1$,

$$
\begin{aligned}
& \left(\int_{2^{k}|z-y| \leq|x-y|<2^{k+1}|z-y|}(|K(x, y)-K(x, z)|+|K(y, x)-K(z, x)|)^{q} d y\right)^{1 / q} \\
& \quad \leq C_{k}\left(2^{k}|z-y|\right)^{-n / q^{\prime}}
\end{aligned}
$$

where $1<q^{\prime}<2$ and $1 / q+1 / q^{\prime}=1$. Moreover, let $m$ be a positive integer and $b$ be a function on $R^{n}$. Set

$$
R_{m+1}(b ; x, y)=b(x)-\sum_{|\alpha| \leq m} \frac{1}{\alpha !} D^{\alpha} b(y)(x-y)^{\alpha} .
$$


The multilinear operator related to the operator $T$ is defined by

$$
T^{b}(f)(x)=\int_{R^{n}} \frac{R_{m+1}(b ; x, y)}{|x-y|^{m}} K(x, y) f(y) d y .
$$

Note that the classical Calderón-Zygmund singular integral operator satisfies Definition 1 (see $[1-3,5,6])$ and that the commutator $[b, T](f)=b T(f)-T(b f)$ is a particular operator of the multilinear operator $T^{b}$ if $m=0$. The multilinear operator $T^{b}$ is a nontrivial generalization of the commutator. It is well known that commutators and multilinear operators are of great interest in harmonic analysis and have been widely studied by many authors (see [21-23]). The main purpose of this paper is to prove the sharp maximal inequalities for the multilinear operator $T^{b}$. As application, we obtain the weighted $L^{p}$-norm inequality and Morrey space boundedness for the multilinear operator $T^{b}$.

\section{Theorems and lemmas}

We shall prove the following theorems.

Theorem 1 Let $T$ be a singular integral operator as in Definition 2 , the sequence $\left\{k C_{k}\right\} \in l^{1}$, $w \in A_{1}, 0<\eta<1, q^{\prime}<r<\infty$ and $D^{\alpha} b \in B M O(w)$ for all $\alpha$ with $|\alpha|=m$. Then there exists a constant $C>0$ such that, for any $f \in C_{0}^{\infty}\left(R^{n}\right)$ and $\tilde{x} \in R^{n}$,

$$
M_{\eta}^{\#}\left(T^{b}(f)\right)(\tilde{x}) \leq C \sum_{|\alpha|=m}\left\|D^{\alpha} b\right\|_{B M O(w)} w(\tilde{x}) M_{r, w}(f)(\tilde{x}) .
$$

Theorem 2 Let $T$ be a singular integral operator as in Definition 2 , the sequence $\left\{k C_{k}\right\} \in l^{1}$, $w \in A_{1}, 0<\eta<1, q^{\prime}<r<\infty, 0<\beta<1$ and $D^{\alpha} b \in \operatorname{Lip}_{\beta}(w)$ for all $\alpha$ with $|\alpha|=m$. Then there exists a constant $C>0$ such that, for any $f \in C_{0}^{\infty}\left(R^{n}\right)$ and $\tilde{x} \in R^{n}$,

$$
M_{\eta}^{\#}\left(T^{b}(f)\right)(\tilde{x}) \leq C \sum_{|\alpha|=m}\left\|D^{\alpha} b\right\|_{L i p_{\beta}(w)} w(\tilde{x}) M_{\beta, r, w}(f)(\tilde{x}) .
$$

Theorem 3 Let $T$ be a singular integral operator as in Definition 2 , the sequence $\left\{k C_{k}\right\} \in l^{1}$, $w \in A_{1}, q^{\prime}<u<\infty$ and $D^{\alpha} b \in B M O(w)$ for all $\alpha$ with $|\alpha|=m$. Then $T^{b}$ is bounded from $L^{u}\left(R^{n}, w\right)$ to $L^{u}\left(R^{n}, w^{1-u}\right)$.

Theorem 4 Let $T$ be a singular integral operator as in Definition 2 , the sequence $\left\{k C_{k}\right\} \in l^{1}$, $w \in A_{1}, q^{\prime}<u<\infty, 0<D<2^{n}$ and $D^{\alpha} b \in B M O(w)$ for all $\alpha$ with $|\alpha|=m$. Then $T^{b}$ is bounded from $L^{u, \varphi}\left(R^{n}, w\right)$ to $L^{u, \varphi}\left(R^{n}, w^{1-u}\right)$.

Theorem 5 Let T be a singular integral operator as in Definition 2 , the sequence $\left\{k C_{k}\right\} \in l^{1}$, $w \in A_{1}, 0<\beta<1, q^{\prime}<u<n / \beta, 1 / v=1 / u-\beta / n$ and $D^{\alpha} b \in \operatorname{Lip}_{\beta}(w)$ for all $\alpha$ with $|\alpha|=m$. Then $T^{b}$ is bounded from $L^{u}\left(R^{n}, w\right)$ to $L^{\nu}\left(R^{n}, w^{1-v}\right)$.

Theorem 6 Let T be a singular integral operator as in Definition 2, the sequence $\left\{k C_{k}\right\} \in l^{1}$, $w \in A_{1}, 0<\beta<1,0<D<2^{n}, q^{\prime}<u<n / \beta, 1 / v=1 / u-\beta / n$ and $D^{\alpha} b \in \operatorname{Lip}_{\beta}(w)$ for all $\alpha$ with $|\alpha|=m$. Then $T^{b}$ is bounded from $L^{u, \varphi}\left(R^{n}, w\right)$ to $L^{v, \varphi}\left(R^{n}, w^{1-v}\right)$.

To prove the theorems, we need the following lemmas. 
Lemma 1 (see [1, p.485]) Let $0<u<v<\infty$ and for any function $f \geq 0$, we define that, for $1 / r=1 / u-1 / v$,

$$
\|f\|_{W L^{v}}=\sup _{\lambda>0} \lambda\left|\left\{x \in R^{n}: f(x)>\lambda\right\}\right|^{1 / v}, \quad N_{u, v}(f)=\sup _{Q}\left\|f \chi_{Q}\right\|_{L^{u}} /\left\|\chi_{Q}\right\|_{L^{r}}
$$

where the sup is taken for all measurable sets $Q$ with $0<|Q|<\infty$. Then

$$
\|f\|_{W L^{v}} \leq N_{u, v}(f) \leq(v /(v-u))^{1 / u}\|f\|_{W L^{\nu}} .
$$

Lemma 2 (see [12]) Let $T$ be a singular integral operator as in Definition 2, the sequence $\left\{C_{k}\right\} \in l^{1}$. Then $T$ is bounded on $L^{p}\left(R^{n}, w\right)$ for $w \in A_{p}$ with $1<p<\infty$, and weak $\left(L^{1}, L^{1}\right)$ bounded.

Lemma 3 (see $[1,7]$ ) Let $0 \leq \eta<n, 1 \leq s<u<n / \eta, 1 / v=1 / u-\eta / n$ and $w \in A_{1}$. Then

$$
\left\|M_{\eta, s, w}(f)\right\|_{L^{v}(w)} \leq C\|f\|_{L^{u}(w)}
$$

Lemma 4 (see [1]) Let $0<p, \eta<\infty$ and $w \in \bigcup_{1 \leq r<\infty} A_{r}$. Then, for any smooth function $f$ for which the left-hand side is finite,

$$
\int_{R^{n}} M_{\eta}(f)(x)^{p} w(x) d x \leq C \int_{R^{n}} M_{\eta}^{\#}(f)(x)^{p} w(x) d x .
$$

Lemma 5 (see [17, 20]) Let $0<p<\infty, 0<\eta<\infty, 0<D<2^{n}$ and $w \in A_{1}$. Then, for any smooth function $f$ for which the left-hand side is finite,

$$
\left\|M_{\eta}(f)\right\|_{L^{p, \varphi}(w)} \leq C\left\|M_{\eta}^{\#}(f)\right\|_{L^{p, \varphi}(w)} .
$$

Lemma 6 (see $[17,20])$ Let $0 \leq \eta<n, 0<D<2^{n}, 1 \leq s<u<n / \eta, 1 / v=1 / u-\eta / n$ and $w \in A_{1}$. Then

$$
\left\|M_{\eta, s, w}(f)\right\|_{L^{\nu, \varphi}(w)} \leq C\|f\|_{L^{u, \varphi}(w)} .
$$

Lemma 7 (see [22]) Let $b$ be a function on $R^{n}$ and $D^{\alpha} A \in L^{u}\left(R^{n}\right)$ for all $\alpha$ with $|\alpha|=m$ and any $u>n$. Then

$$
\left|R_{m}(b ; x, y)\right| \leq C|x-y|^{m} \sum_{|\alpha|=m}\left(\frac{1}{|\tilde{Q}(x, y)|} \int_{\tilde{Q}(x, y)}\left|D^{\alpha} b(z)\right|^{u} d z\right)^{1 / u},
$$

where $\tilde{Q}$ is the cube centered at $x$ and having side length $5 \sqrt{n}|x-y|$.

\section{Proofs of theorems}

Proof of Theorem 1 It suffices to prove for $f \in C_{0}^{\infty}\left(R^{n}\right)$ and some constant $C_{0}$ that the following inequality holds:

$$
\left(\frac{1}{|Q|} \int_{Q}\left|T^{b}(f)(x)-C_{0}\right|^{\eta} d x\right)^{1 / \eta} \leq C \sum_{|\alpha|=m}\left\|D^{\alpha} b\right\|_{B M O(w)} w(\tilde{x}) M_{r, w}(f)(\tilde{x}) .
$$


Fix a cube $Q=Q\left(x_{0}, d\right)$ and $\tilde{x} \in Q$. Let $\tilde{Q}=5 \sqrt{n} Q$ and $\tilde{b}(x)=b(x)-\sum_{|\alpha|=m} \frac{1}{\alpha !}\left(D^{\alpha} b\right)_{\tilde{Q}} x^{\alpha}$, then $R_{m}(b ; x, y)=R_{m}(\tilde{b} ; x, y)$ and $D^{\alpha} \tilde{b}=D^{\alpha} b-\left(D^{\alpha} b\right)_{\tilde{Q}}$ for $|\alpha|=m$. We write, for $f_{1}=f \chi_{\tilde{Q}}$ and $f_{2}=f \chi_{R^{n} \backslash \tilde{Q}}$,

$$
\begin{aligned}
T^{b}(f)(x)= & \int_{R^{n}} \frac{R_{m}(\tilde{b} ; x, y)}{|x-y|^{m}} K(x, y) f_{1}(y) d y \\
& -\sum_{|\alpha|=m} \frac{1}{\alpha !} \int_{R^{n}} \frac{(x-y)^{\alpha} D^{\alpha} \tilde{b}(y)}{|x-y|^{m}} K(x, y) f_{1}(y) d y \\
& +\int_{R^{n}} \frac{R_{m+1}(\tilde{b} ; x, y)}{|x-y|^{m}} K(x, y) f_{2}(y) d y \\
= & T\left(\frac{R_{m}(\tilde{b} ; x, \cdot)}{|x-\cdot|^{m}} f_{1}\right)-T\left(\sum_{|\alpha|=m} \frac{1}{\alpha !} \frac{(x-\cdot)^{\alpha} D^{\alpha} \tilde{b}}{|x-\cdot|^{m}} f_{1}\right)+T^{\tilde{b}}\left(f_{2}\right)(x),
\end{aligned}
$$

then

$$
\begin{aligned}
& \left(\frac{1}{|Q|} \int_{Q}\left|T^{b}(f)(x)-T^{\tilde{b}}\left(f_{2}\right)\left(x_{0}\right)\right|^{\eta} d x\right)^{1 / \eta} \\
& \leq C\left(\frac{1}{|Q|} \int_{Q}\left|T\left(\frac{R_{m}(\tilde{b} ; x, \cdot)}{|x-\cdot|^{m}} f_{1}\right)\right|^{\eta} d x\right)^{1 / \eta} \\
& \quad+C\left(\frac{1}{|Q|} \int_{Q}\left|T\left(\sum_{|\alpha|=m} \frac{(x-\cdot)^{\alpha} D^{\alpha} \tilde{b}}{|x-\cdot|^{m}} f_{1}\right)\right|^{\eta} d x\right)^{1 / \eta} \\
& \quad+C\left(\frac{1}{|Q|} \int_{Q}\left|T^{\tilde{b}}\left(f_{2}\right)(x)-T^{\tilde{b}}\left(f_{2}\right)\left(x_{0}\right)\right|^{\eta} d x\right)^{1 / \eta} \\
& =I_{1}+I_{2}+I_{3} .
\end{aligned}
$$

For $I_{1}$, noting that $w \in A_{1}, w$ satisfies the reverse of Hölder's inequality,

$$
\left(\frac{1}{|Q|} \int_{Q} w(x)^{p_{0}} d x\right)^{1 / p_{0}} \leq \frac{C}{|Q|} \int_{Q} w(x) d x
$$

for all cube $Q$ and some $1<p_{0}<\infty$ (see [1]). We take $u=r p_{0} /\left(r+p_{0}-1\right)$ in Lemma 7 and have $1<u<r$ and $p_{0}=u(r-1) /(r-u)$. Then by Lemma 7 and Hölder's inequality, we get

$$
\begin{aligned}
& \left|R_{m}(\tilde{b} ; x, y)\right| \\
& \leq C|x-y|^{m} \sum_{|\alpha|=m}\left(\frac{1}{|\tilde{Q}(x, y)|} \int_{\tilde{Q}(x, y)}\left|D^{\alpha} \tilde{b}(z)\right|^{u} d z\right)^{1 / u} \\
& \leq C|x-y|^{m} \sum_{|\alpha|=m}|\tilde{Q}|^{-1 / u}\left(\int_{\tilde{Q}(x, y)}\left|D^{\alpha} \tilde{b}(z)\right|^{u} w(z)^{u(1-r) / r} w(z)^{u(r-1) / r} d z\right)^{1 / u} \\
& \leq C|x-y|^{m} \sum_{|\alpha|=m}|\tilde{Q}|^{-1 / u}\left(\int_{\tilde{Q}(x, y)}\left|D^{\alpha} \tilde{b}(z)\right|^{r} w(z)^{1-r} d z\right)^{1 / r} \\
& \quad \times\left(\int_{\tilde{Q}(x, y)} w(z)^{u(r-1) /(r-u)} d z\right)^{(r-u) / r u}
\end{aligned}
$$




$$
\begin{aligned}
\leq & C|x-y|^{m} \sum_{|\alpha|=m}|\tilde{Q}|^{-1 / u}\left\|D^{\alpha} b\right\|_{B M O(w)} w(\tilde{Q})^{1 / r}|\tilde{Q}|^{(r-u) / r u} \\
& \times\left(\frac{1}{|\tilde{Q}(x, y)|} \int_{\tilde{Q}(x, y)} w(z)^{p_{0}} d z\right)^{(r-u) / r u} \\
\leq & C|x-y|^{m} \sum_{|\alpha|=m}\left\|D^{\alpha} b\right\|_{B M O(w)}|\tilde{Q}|^{-1 / u} w(\tilde{Q})^{1 / r}|\tilde{Q}|^{1 / u-1 / r}\left(\frac{1}{|\tilde{Q}(x, y)|} \int_{\tilde{Q}(x, y)} w(z) d z\right)^{(r-1) / r} \\
\leq & C|x-y|^{m} \sum_{|\alpha|=m}\left\|D^{\alpha} b\right\|_{B M O(w)}|\tilde{Q}|^{-1 / u} w(\tilde{Q})^{1 / r}|\tilde{Q}|^{1 / u-1 / r} w(\tilde{Q})^{1-1 / r}|\tilde{Q}|^{1 / r-1} \\
\leq & C|x-y|^{m} \sum_{|\alpha|=m}\left\|D^{\alpha} b\right\|_{B M O(w)} \frac{w(\tilde{Q})}{|\tilde{Q}|} \\
\leq C|x-y|^{m} & \sum_{|\alpha|=m}\left\|D^{\alpha} b\right\|_{B M O(w)} w(\tilde{x}) .
\end{aligned}
$$

Thus, by the $L^{s}$-boundedness of $T$ (see Lemma 2) for $1<s<r$ and $w \in A_{1} \subseteq A_{r / s}$, we obtain

$$
\begin{aligned}
I_{1} \leq & \frac{C}{|Q|} \int_{Q}\left|T\left(\frac{R_{m}(\tilde{b} ; x, \cdot)}{|x-\cdot|^{m}} f_{1}\right)\right| d x \\
\leq & C \sum_{|\alpha|=m}\left\|D^{\alpha} b\right\|_{B M O(w)} w(\tilde{x})\left(\frac{1}{|Q|} \int_{R^{n}}\left|T\left(f_{1}\right)(x)\right|^{s} d x\right)^{1 / s} \\
\leq & C \sum_{|\alpha|=m}\left\|D^{\alpha} b\right\|_{B M O(w)} w(\tilde{x})|Q|^{-1 / s}\left(\int_{R^{n}}\left|f_{1}(x)\right|^{s} d x\right)^{1 / s} \\
\leq & C \sum_{|\alpha|=m}\left\|D^{\alpha} b\right\|_{B M O(w)} w(\tilde{x})|Q|^{-1 / s}\left(\int_{\tilde{Q}}|f(x)|^{s} w(x)^{s / r} w(x)^{-s / r} d x\right)^{1 / s} \\
\leq & C \sum_{|\alpha|=m}\left\|D^{\alpha} b\right\|_{B M O(w)} w(\tilde{x})|Q|^{-1 / s}\left(\int_{\tilde{Q}}|f(x)|^{r} w(x) d x\right)^{1 / r}\left(\int_{\tilde{Q}} w(x)^{-s /(r-s)} d x\right)^{(r-s) / r s} \\
\leq & C \sum_{|\alpha|=m}\left\|D^{\alpha} b\right\|_{B M O(w)} w(\tilde{x})|Q|^{-1 / s} w(\tilde{Q})^{1 / r}\left(\frac{1}{w(\tilde{Q})} \int_{\tilde{Q}}|f(x)|^{r} w(x) d x\right)^{1 / r} \\
& \times\left(\frac{1}{|\tilde{Q}|} \int_{\tilde{Q}} w(x)^{-s /(r-s)} d x\right)^{(r-s) / r s}\left(\frac{1}{|\tilde{Q}|} \int_{\tilde{Q}} w(x) d x\right)^{1 / r}|\tilde{Q}|^{1 / s} w(\tilde{Q})^{-1 / r} \\
\leq & C \sum_{|\alpha|=m}\left\|D^{\alpha} b\right\|_{B M O(w)} w(\tilde{x}) M_{r, w}(f)(\tilde{x}) .
\end{aligned}
$$

For $I_{2}$, by the weak $\left(L^{1}, L^{1}\right)$ boundedness of $T$ (see Lemma 2 ) and Kolmogoro's inequality (see Lemma 1), we obtain

$$
\begin{aligned}
I_{2} & \leq C \sum_{|\alpha|=m}\left(\frac{1}{|Q|} \int_{Q}\left|T\left(D^{\alpha} \tilde{b f}_{1}\right)(x)\right|^{\eta} d x\right)^{1 / \eta} \\
& \leq C \sum_{|\alpha|=m} \frac{|Q|^{1 / \eta-1}}{|Q|^{1 / \eta}} \frac{\left\|T\left(D^{\alpha} \tilde{b f}_{1}\right) \chi_{Q}\right\|_{L^{\eta}}}{\left\|\chi_{Q}\right\|_{L^{\eta /(1-\eta)}}} \\
& \leq C \sum_{|\alpha|=m} \frac{1}{|Q|}\left\|T\left(D^{\alpha} \tilde{b f_{1}}\right)\right\|_{W L^{1}}
\end{aligned}
$$


Fens Journal of Inequalities and Applications 2014, 2014:188

Page 8 of 17

http://www.journalofinequalitiesandapplications.com/content/2014/1/188

$$
\begin{aligned}
& \leq C \sum_{|\alpha|=m} \frac{1}{|Q|} \int_{R^{n}}\left|D^{\alpha} \tilde{b}(x) f_{1}(x)\right| d x \\
& \leq C \sum_{|\alpha|=m} \frac{1}{|Q|} \int_{\tilde{Q}}\left|D^{\alpha} b(x)-\left(D^{\alpha} b\right)_{\tilde{Q}}\right| w(x)^{-1 / r}|f(x)| w(x)^{1 / r} d x \\
& \leq C \sum_{|\alpha|=m} \frac{1}{|Q|}\left(\int_{\tilde{Q}}\left|\left(D^{\alpha} b(x)-\left(D^{\alpha} b\right)_{\tilde{Q}}\right)\right|^{r^{\prime}} w(x)^{1-r^{\prime}} d x\right)^{1 / r^{\prime}}\left(\int_{\tilde{Q}}|f(x)|^{r} w(x) d x\right)^{1 / r} \\
& \leq C \sum_{|\alpha|=m} \frac{1}{|Q|}\left\|D^{\alpha} b\right\|_{B M O(w)} w(\tilde{Q})^{1 / r^{\prime}} w(\tilde{Q})^{1 / r}\left(\frac{1}{w(\tilde{Q})} \int_{\tilde{Q}}|f(x)|^{r} w(x) d x\right)^{1 / r} \\
& \leq C \sum_{|\alpha|=m}\left\|D^{\alpha} b\right\|_{B M O(w)} \frac{w(\tilde{Q})}{|\tilde{Q}|} M_{r, w}(f)(\tilde{x}) \\
& \leq C \sum_{|\alpha|=m}\left\|D^{\alpha} b\right\|_{B M O(w)} w(\tilde{x}) M_{r, w}(f)(\tilde{x}) .
\end{aligned}
$$

For $I_{3}$, noting that $|x-y| \approx\left|x_{0}-y\right|$ for $x \in Q$ and $y \in R^{n} \backslash Q$, we write

$$
\begin{aligned}
& \left|T^{\tilde{b}}\left(f_{2}\right)(x)-T^{\tilde{b}}\left(f_{2}\right)\left(x_{0}\right)\right| \\
& \leq \int_{R^{n}}\left|R_{m}(\tilde{b} ; x, y)-R_{m}\left(\tilde{b} ; x_{0}, y\right)\right| \frac{|K(x, y)|}{|x-y|^{m}}\left|f_{2}(y)\right| d y \\
& \quad+\int_{R^{n}}\left|\frac{K(x, y)}{|x-y|^{m}}-\frac{K\left(x_{0}, y\right)}{\left|x_{0}-y\right|^{m}}\right|\left|R_{m}\left(\tilde{b} ; x_{0}, y\right)\right|\left|f_{2}(y)\right| d y \\
& \quad+\sum_{|\alpha|=m} \frac{1}{\alpha !} \int_{R^{n}}\left|\frac{K(x, y)(x-y)^{\alpha}}{|x-y|^{m}}-\frac{K\left(x_{0}, y\right)\left(x_{0}-y\right)^{\alpha}}{\left|x_{0}-y\right|^{m}}\right|\left|D^{\alpha} \tilde{b}(y)\right|\left|f_{2}(y)\right| d y \\
& =I_{3}^{(1)}(x)+I_{3}^{(2)}(x)+I_{3}^{(3)}(x) .
\end{aligned}
$$

For $I_{3}^{(1)}(x)$, by the formula (see [22])

$$
R_{m}(\tilde{b} ; x, y)-R_{m}\left(\tilde{b} ; x_{0}, y\right)=\sum_{|\gamma|<m} \frac{1}{\gamma !} R_{m-|\gamma|}\left(D^{\gamma} \tilde{b} ; x, x_{0}\right)(x-y)^{\gamma}
$$

and Lemma 7, we have, similar to the proof of $I_{1}$,

$$
\left|R_{m}(\tilde{b} ; x, y)-R_{m}\left(\tilde{b} ; x_{0}, y\right)\right| \leq C \sum_{|\gamma|<m} \sum_{|\alpha|=m}\left|x-x_{0}\right|^{m-|\gamma|}|x-y|^{|\gamma|}\left\|D^{\alpha} b\right\|_{B M O(w)} w(\tilde{x})
$$

and

$$
\left|R_{m}\left(\tilde{b} ; x_{0}, y\right)\right| \leq C \sum_{|\alpha|=m}\left|x-x_{0}\right|^{m}\left\|D^{\alpha} b\right\|_{B M O(w)} w(\tilde{x})
$$

Thus, by $w \in A_{1} \subseteq A_{r}$, we get

$$
\begin{aligned}
I_{3}^{(1)}(x) & \leq \sum_{k=0}^{\infty} \int_{2^{k+1} \tilde{Q} \backslash 2^{k} \tilde{Q}}\left|R_{m}(\tilde{b} ; x, y)-R_{m}\left(\tilde{b} ; x_{0}, y\right)\right| \frac{|K(x, y)|}{|x-y|^{m}}|f(y)| d y \\
& \leq C \sum_{|\alpha|=m}\left\|D^{\alpha} b\right\|_{B M O(w)} w(\tilde{x}) \sum_{k=0}^{\infty} \int_{2^{k+1} \tilde{Q} \backslash 2^{k} \tilde{Q}} \frac{\left|x-x_{0}\right|}{\left|x_{0}-y\right|^{n+1}}|f(y)| d y
\end{aligned}
$$


Fen Journal of Inequalities and Applications 2014, 2014:188

Page 9 of 17

http://www.journalofinequalitiesandapplications.com/content/2014/1/188

$$
\begin{aligned}
\leq & C \sum_{|\alpha|=m}\left\|D^{\alpha} b\right\|_{B M O(w)} w(\tilde{x}) \sum_{k=1}^{\infty} \frac{d}{\left(2^{k} d\right)^{n+1}} \int_{2^{k} \tilde{Q}}|f(y)| w(y)^{1 / r} w(y)^{-1 / r} d y \\
\leq & C \sum_{|\alpha|=m}\left\|D^{\alpha} b\right\|_{B M O(w)} w(\tilde{x}) \sum_{k=1}^{\infty} \frac{d}{\left(2^{k} d\right)^{n+1}}\left(\int_{2^{k} \tilde{Q}}|f(y)|^{r} w(y) d y\right)^{1 / r} \\
& \times\left(\int_{2^{k} \tilde{Q}} w(y)^{-1 /(r-1)} d y\right)^{(r-1) / r} \\
\leq & C \sum_{|\alpha|=m}\left\|D^{\alpha} b\right\|_{B M O(w)} w(\tilde{x}) \sum_{k=1}^{\infty} \frac{d}{\left(2^{k} d\right)^{n+1}} w\left(2^{k} \tilde{Q}\right)^{1 / r} \\
& \times\left(\frac{1}{w\left(2^{k} \tilde{Q}\right)} \int_{2^{k} \tilde{Q}}|f(y)|^{r} w(y) d x\right)^{1 / r}\left(\frac{1}{\left|2^{k} \tilde{Q}\right|} \int_{2^{k} \tilde{Q}} w(y)^{-1 /(r-1)} d y\right)^{(r-1) / r} \\
& \times\left(\frac{1}{\left|2^{k} \tilde{Q}\right|} \int_{2^{k} \tilde{Q}} w(y) d y\right)^{1 / r}\left|2^{k} \tilde{Q}\right| w\left(2^{k} \tilde{Q}\right)^{-1 / r} \\
\leq & C \sum_{|\alpha|=m}\left\|D^{\alpha} b\right\|_{B M O(w)} w(\tilde{x}) M_{r, w}(f)(\tilde{x}) \sum_{k=1}^{\infty} 2^{-k} \\
\leq & C \sum_{|\alpha|=m}\left\|D^{\alpha} b\right\|_{B M O(w)} w(\tilde{x}) M_{r, w}(f)(\tilde{x}) .
\end{aligned}
$$

For $I_{3}^{(2)}(x)$, we take $1<p<\infty$ such that $1 / p+1 / q+1 / r=1$. Recalling $r>q^{\prime}$ and $w \in A_{1} \subseteq$ $A_{r / p+1}$, we get

$$
\begin{aligned}
& I_{3}^{(2)}(x) \leq \sum_{k=0}^{\infty} \int_{2^{k+1} \tilde{Q} \backslash 2^{k} \tilde{Q}}\left|K(x, y)-K\left(x_{0}, y\right)\right| \frac{\left|R_{m}\left(\tilde{b} ; x_{0}, y\right)\right|}{|x-y|^{m}}|f(y)| w(y)^{1 / r} w(y)^{-1 / r} d y \\
& +\int_{2^{k+1} \tilde{Q} \backslash 2^{k} \tilde{Q}}\left|\frac{1}{|x-y|^{m}}-\frac{1}{\left|x_{0}-y\right|^{m}}\right|\left|K\left(x_{0}, y\right)\right|\left|R_{m}\left(\tilde{b} ; x_{0}, y\right)\right||f(y)| d y \\
& \leq C \sum_{|\alpha|=m}\left\|D^{\alpha} b\right\|_{B M O(w)} w(\tilde{x}) \sum_{k=0}^{\infty}\left(\int_{2^{k+1} \tilde{Q} \backslash 2^{k} \tilde{Q}}\left|K(x, y)-K\left(x_{0}, y\right)\right|^{q} d y\right)^{1 / q} \\
& \times\left(\int_{2^{k+1} \tilde{Q} \backslash 2^{k} \tilde{Q}}|f(y)|^{r} w(y) d y\right)^{1 / r}\left(\int_{2^{k+1} \tilde{Q} \backslash 2^{k} \tilde{Q}} w(y)^{-p / r} d y\right)^{1 / p} \\
& +C \sum_{|\alpha|=m}\left\|D^{\alpha} b\right\|_{B M O(w)} w(\tilde{x}) \sum_{k=0}^{\infty} \int_{2^{k+1} \tilde{Q} \backslash 2^{k} \tilde{Q}} \frac{\left|x-x_{0}\right|}{\left|x_{0}-y\right|^{n+1}}|f(y)| w(y)^{1 / r} w(y)^{-1 / r} d y \\
& \leq C \sum_{|\alpha|=m}\left\|D^{\alpha} b\right\|_{B M O(w)} w(\tilde{x}) \sum_{k=1}^{\infty} C_{k}\left|2^{k} Q\right|^{-1 / q^{\prime}} w\left(2^{k} \tilde{Q}\right)^{1 / r} \\
& \times\left(\frac{1}{w\left(2^{k} \tilde{Q}\right)} \int_{2^{k} \tilde{Q}}|f(y)|^{r} w(y) d y\right)^{1 / r}\left(\frac{1}{\left|2^{k} \tilde{Q}\right|} \int_{2^{k} \tilde{Q}} w(y)^{-p / r} d y\right)^{1 / p} \\
& \times\left(\frac{1}{\left|2^{k} \tilde{Q}\right|} \int_{2^{k} \tilde{Q}} w(y) d y\right)^{1 / r}\left|2^{k} \tilde{Q}\right|^{1 / r+1 / p} w\left(2^{k} \tilde{Q}\right)^{-1 / r} \\
& +C \sum_{|\alpha|=m}\left\|D^{\alpha} b\right\|_{B M O(w)} w(\tilde{x}) \sum_{k=1}^{\infty} \frac{d}{\left(2^{k} d\right)^{n+1}} \\
& \times\left(\int_{2^{k} \tilde{Q}}|f(y)|^{r} w(y) d y\right)^{1 / r}\left(\int_{2^{k} \tilde{Q}} w(y)^{-1 /(r-1)} d y\right)^{(r-1) / r}
\end{aligned}
$$




$$
\begin{aligned}
& \leq C \sum_{|\alpha|=m}\left\|D^{\alpha} b\right\|_{B M O(w)} w(\tilde{x}) \sum_{k=1}^{\infty}\left(C_{k}+2^{-k}\right)\left(\frac{1}{w\left(2^{k} \tilde{Q}\right)} \int_{2^{k} \tilde{Q}}|f(y)|^{r} w(y) d y\right)^{1 / r} \\
& \leq C \sum_{|\alpha|=m}\left\|D^{\alpha} b\right\|_{B M O(w)} w(\tilde{x}) M_{r, w}(f)(\tilde{x}) .
\end{aligned}
$$

Similarly, we have, for $r<p_{1}^{\prime}<\infty, 1<s_{1}, s_{2}<\infty$ with $1 / p_{1}+1 / q+1 / r+1 / s_{1}=1$ and $1 / q+$ $1 / r+1 / s_{2}=1$,

$$
\begin{aligned}
& I_{3}^{(3)}(x) \leq \sum_{|\alpha|=m} \sum_{k=0}^{\infty} \int_{2^{k+1} \tilde{Q} \backslash 2^{k} \tilde{Q}}\left|K(x, y)-K\left(x_{0}, y\right)\right| \frac{\left|(x-y)^{\alpha}\right|}{|x-y|^{m}} \\
& \times\left|D^{\alpha} b(y)-\left(D^{\alpha} b\right)_{2^{k+1} \tilde{Q}}\right| w(y)^{\left(1-p_{1}\right) / p_{1}}|f(y)| w(y)^{1 / r} w(y)^{\left(p_{1}-1\right) / p_{1}-1 / r} d y \\
& +\sum_{|\alpha|=m} \sum_{k=0}^{\infty} \int_{2^{k+1} \tilde{Q} \backslash 2^{k} \tilde{Q}}\left|K(x, y)-K\left(x_{0}, y\right)\right| \frac{\left|(x-y)^{\alpha}\right|}{|x-y|^{m}} \\
& \times\left|\left(D^{\alpha} b\right)_{2^{k+1} \tilde{Q}}-\left(D^{\alpha} b\right)_{\tilde{Q}}\right||f(y)| w(y)^{1 / r} w(y)^{-1 / r} d y \\
& +\sum_{|\alpha|=m} \int_{2^{k+1} \tilde{Q} \backslash 2^{k} \tilde{Q}}\left|\frac{(x-y)^{\alpha}}{|x-y|^{m}}-\frac{\left(x_{0}-y\right)^{\alpha}}{\left|x_{0}-y\right|^{m}}\right|\left|K\left(x_{0}, y\right)\right||f(y)|\left|D^{\alpha} \tilde{b}(y)\right| d y \\
& \leq C \sum_{|\alpha|=m} \sum_{k=0}^{\infty}\left(\int_{2^{k+1} \tilde{Q} \mid 2^{k} \tilde{Q}}\left|K(x, y)-K\left(x_{0}, y\right)\right|^{q} d y\right)^{1 / q}\left(\int_{2^{k+1} \tilde{Q}}|f(y)|^{r} w(y) d y\right)^{1 / r} \\
& \times\left(\int_{2^{k+1} \tilde{Q}}\left|D^{\alpha} b(y)-\left(D^{\alpha} b\right)_{2^{k+1} \tilde{Q}}\right|^{p_{1}} w(y)^{1-p_{1}} d y\right)^{1 / p_{1}} \\
& \times\left(\int_{2^{k+1} \tilde{Q}} w(y)^{-\left(1 / r-1 / p_{1}^{\prime}\right) s_{1}} d y\right)^{1 / s_{1}} \\
& +C \sum_{|\alpha|=m} \sum_{k=0}^{\infty}\left(\int_{2^{k+1} \tilde{Q} \backslash 2^{k} \tilde{Q}}\left|K(x, y)-K\left(x_{0}, y\right)\right|^{q} d y\right)^{1 / q} \\
& \times\left(\int_{2^{k+1} \tilde{Q}}|f(y)|^{r} w(y) d y\right)^{1 / r} \\
& \times\left|\left(D^{\alpha} b\right)_{2^{k+1} \tilde{Q}}-\left(D^{\alpha} b\right)_{\tilde{Q}}\right|\left(\int_{2^{k+1} \tilde{Q}} w(y)^{-s_{2} / r} d y\right)^{1 / s_{2}} \\
& +C \sum_{|\alpha|=m} \sum_{k=0}^{\infty} \int_{2^{k+1} \tilde{Q} \backslash 2^{k} \tilde{Q}} \frac{\left|x-x_{0}\right|}{\left|x_{0}-y\right|^{n+1}} \\
& \times|f(y)|\left|D^{\alpha} b(y)-\left(D^{\alpha} b\right){ }_{2^{k+1} \tilde{Q}}\right| w(y)^{1 / r} w(y)^{-1 / r} d y \\
& +C \sum_{|\alpha|=m} \sum_{k=0}^{\infty} \int_{2^{k+1} \tilde{Q} \backslash 2^{k} \tilde{Q}} \frac{\left|x-x_{0}\right|}{\left|x_{0}-y\right|^{n+1}} \\
& \times|f(y)|\left|\left(D^{\alpha} b\right)_{2^{k+1} \tilde{Q}}-\left(D^{\alpha} b\right)_{\tilde{Q}}\right| w(y)^{1 / r} w(y)^{-1 / r} d y \\
& \leq C \sum_{|\alpha|=m} \sum_{k=1}^{\infty} C_{k}\left|2^{k} Q\right|^{-1 / q^{\prime}}\left\|D^{\alpha} b\right\|_{B M O(w)} w\left(2^{k} \tilde{Q}\right)^{1 / p_{1}} w\left(2^{k} \tilde{Q}\right)^{1 / r} \\
& \times\left(\frac{1}{w\left(2^{k} \tilde{Q}\right)} \int_{2^{k} \tilde{Q}}|f(y)|^{r} w(y) d y\right)^{1 / r}
\end{aligned}
$$




$$
\begin{aligned}
& \times\left(\frac{1}{\left|2^{k} \tilde{Q}\right|} \int_{2^{k} \tilde{Q}} w(y) d y\right)^{1 / r-1 / p_{1}^{\prime}}\left(\frac{1}{\left|2^{k} \tilde{Q}\right|} \int_{2^{k} \tilde{Q}} w(y)^{-\left(1 / r-1 / p_{1}^{\prime}\right) s_{1}} d y\right)^{1 / s_{1}} \\
& \times\left|2^{k} \tilde{Q}\right|^{1 / s_{1}+1 / r-1 / p_{1}^{\prime}} w\left(2^{k} \tilde{Q}\right)^{-1 / r+1 / p_{1}^{\prime}} \\
& +C \sum_{|\alpha|=m} \sum_{k=1}^{\infty} C_{k}\left|2^{k} Q\right|^{-1 / q^{\prime}} k\left\|D^{\alpha} b\right\|_{B M O(w)} w(\tilde{x}) w\left(2^{k} \tilde{Q}\right)^{1 / r} \\
& \times\left(\frac{1}{w\left(2^{k} \tilde{Q}\right)} \int_{2^{k} \tilde{Q}}|f(y)|^{r} w(y) d y\right)^{1 / r}\left(\frac{1}{\left|2^{k} \tilde{Q}\right|} \int_{2^{k} \tilde{Q}} w(y) d y\right)^{1 / r} \\
& \times\left(\frac{1}{\left|2^{k} \tilde{Q}\right|} \int_{2^{k} \tilde{Q}} w(y)^{-s_{2} / r} d y\right)^{1 / s_{2}}\left|2^{k} \tilde{Q}\right|^{1 / s_{2}+1 / r} w\left(2^{k} \tilde{Q}\right)^{-1 / r} \\
& +C \sum_{|\alpha|=m} \sum_{k=1}^{\infty} \frac{d}{\left(2^{k} d\right)^{n+1}}\left(\int_{2^{k} \tilde{Q}}\left|D^{\alpha} b(y)-\left(D^{\alpha} b\right)_{2^{k} \tilde{Q}}\right|^{r^{\prime}} w(y)^{1-r^{\prime}} d y\right)^{1 / r^{\prime}} \\
& \times\left(\int_{2^{k+1} \tilde{Q}}|f(y)|^{r} w(y) d y\right)^{1 / r} \\
& +C \sum_{|\alpha|=m} \sum_{k=1}^{\infty} \frac{d}{\left(2^{k} d\right)^{n+1}} k\left\|D^{\alpha} b\right\|_{B M O(w)} w(\tilde{x})\left(\int_{2^{k} \tilde{Q}} w(y)^{-r^{\prime} / r} d y\right)^{1 / r^{\prime}} \\
& \times\left(\int_{2^{k+1} \tilde{Q}}|f(y)|^{r} w(y) d y\right)^{1 / r} \\
& \leq \sum_{|\alpha|=m}\left\|D^{\alpha} b\right\|_{B M O(w)} \sum_{k=1}^{\infty} C_{k} \frac{w\left(2^{k} \tilde{Q}\right)}{\left|2^{k} \tilde{Q}\right|}\left(\frac{1}{w\left(2^{k} \tilde{Q}\right)} \int_{2^{k} \tilde{Q}}|f(y)|^{r} w(y) d y\right)^{1 / r} \\
& +\sum_{|\alpha|=m}\left\|D^{\alpha} b\right\|_{B M O(w)} w(\tilde{x}) \sum_{k=1}^{\infty} k C_{k}\left(\frac{1}{w\left(2^{k} \tilde{Q}\right)} \int_{2^{k} \tilde{Q}}|f(y)|^{r} w(y) d y\right)^{1 / r} \\
& +C \sum_{|\alpha|=m}\left\|D^{\alpha} b\right\|_{B M O(w)} \sum_{k=1}^{\infty} 2^{-k} \frac{w\left(2^{k} \tilde{Q}\right)}{\left|2^{k} \tilde{Q}\right|}\left(\frac{1}{w\left(2^{k} \tilde{Q}\right)} \int_{2^{k} \tilde{Q}}|f(y)|^{r} w(y) d x\right)^{1 / r} \\
& +C \sum_{|\alpha|=m}\left\|D^{\alpha} b\right\|_{B M O(w)} w(\tilde{x}) \sum_{k=1}^{\infty} k 2^{-k}\left(\frac{1}{w\left(2^{k} \tilde{Q}\right)} \int_{2^{k} \tilde{Q}}|f(y)|^{r} w(y) d x\right)^{1 / r} \\
& \leq C \sum_{|\alpha|=m}\left\|D^{\alpha} b\right\|_{B M O(w)} w(\tilde{x}) M_{r, w}(f)(\tilde{x}) .
\end{aligned}
$$

Thus

$$
I_{3} \leq C \sum_{|\alpha|=m}\left\|D^{\alpha} b\right\|_{B M O(w)} w(\tilde{x}) M_{r, w}(f)(\tilde{x})
$$

These complete the proof of Theorem 1 .

Proof of Theorem 2 It suffices to prove for $f \in C_{0}^{\infty}\left(R^{n}\right)$ and some constant $C_{0}$ that the following inequality holds:

$$
\left(\frac{1}{|Q|} \int_{Q}\left|T^{b}(f)(x)-C_{0}\right|^{\eta} d x\right)^{1 / \eta} \leq C \sum_{|\alpha|=m}\left\|D^{\alpha} b\right\|_{L i p_{\beta}(w)} w(\tilde{x}) M_{\beta, r, w}(f)(\tilde{x}) .
$$


Fens Journal of Inequalities and Applications 2014, 2014:188

Page 12 of 17

http://www.journalofinequalitiesandapplications.com/content/2014/1/188

Fix a cube $Q=Q\left(x_{0}, d\right)$ and $\tilde{x} \in Q$. Similar to the proof of Theorem 1 , we have, for $f_{1}=f \chi_{\tilde{Q}}$ and $f_{2}=f \chi_{R^{n} \backslash \tilde{Q}}$,

$$
\begin{aligned}
& \left(\frac{1}{|Q|} \int_{Q}\left|T^{b}(f)(x)-T^{\tilde{b}}\left(f_{2}\right)\left(x_{0}\right)\right|^{\eta} d x\right)^{1 / \eta} \\
& \leq C\left(\frac{1}{|Q|} \int_{Q}\left|T\left(\frac{R_{m}(\tilde{b} ; x, \cdot)}{|x-\cdot|^{m}} f_{1}\right)\right|^{\eta} d x\right)^{1 / \eta} \\
& \quad+C\left(\frac{1}{|Q|} \int_{Q}\left|T\left(\sum_{|\alpha|=m} \frac{(x-\cdot)^{\alpha} D^{\alpha} \tilde{b}}{|x-\cdot|^{m}} f_{1}\right)\right|^{\eta} d x\right)^{1 / \eta} \\
& \quad+C\left(\frac{1}{|Q|} \int_{Q}\left|T^{\tilde{b}}\left(f_{2}\right)(x)-T^{\tilde{b}}\left(f_{2}\right)\left(x_{0}\right)\right|^{\eta} d x\right)^{1 / \eta} \\
& =J_{1}+J_{2}+J_{3} .
\end{aligned}
$$

For $J_{1}$ and $J_{2}$, by using the same argument as in the proof of Theorem 1, we get

$$
\begin{aligned}
& \left|R_{m}(\tilde{b} ; x, y)\right| \\
& \leq C|x-y|^{m} \sum_{|\alpha|=m}|\tilde{Q}|^{-1 / q}\left(\int_{\tilde{Q}(x, y)}\left|D^{\alpha} \tilde{b}(z)\right|^{q} w(z)^{q(1-r) / r} w(z)^{q(r-1) / r} d z\right)^{1 / q} \\
& \leq C|x-y|^{m} \sum_{|\alpha|=m}|\tilde{Q}|^{-1 / q}\left(\int_{\tilde{Q}(x, y)}\left|D^{\alpha} \tilde{b}(z)\right|^{r} w(z)^{1-r} d z\right)^{1 / r} \\
& \times\left(\int_{\tilde{Q}(x, y)} w(z)^{q(r-1) /(r-q)} d z\right)^{(r-q) / r q} \\
& \leq C|x-y|^{m} \sum_{|\alpha|=m}|\tilde{Q}|^{-1 / q}\left\|D^{\alpha} b\right\|_{\operatorname{Lip}_{\beta}(w)} w(\tilde{Q})^{\beta / n+1 / r}|\tilde{Q}|^{(r-q) / r q} \\
& \times\left(\frac{1}{|\tilde{Q}(x, y)|} \int_{\tilde{Q}(x, y)} w(z)^{p_{0}} d z\right)^{(r-q) / r q}
\end{aligned}
$$

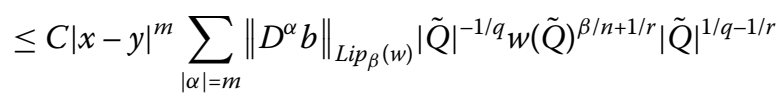

$$
\begin{aligned}
& \times\left(\frac{1}{|\tilde{Q}(x, y)|} \int_{\tilde{Q}(x, y)} w(z) d z\right)^{(r-1) / r}
\end{aligned}
$$

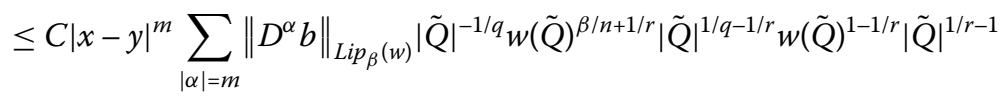

$$
\begin{aligned}
& \leq C|x-y|^{m} \sum_{|\alpha|=m}\left\|D^{\alpha} b\right\|_{\operatorname{Lip}_{\beta}(w)} \frac{w(\tilde{Q})^{\beta / n+1}}{|\tilde{Q}|} \\
& \leq C|x-y|^{m} \sum_{|\alpha|=m}\left\|D^{\alpha} b\right\|_{\operatorname{Lip}_{\beta}(w)} w(\tilde{Q})^{\beta / n} w(\tilde{x}) .
\end{aligned}
$$

Thus

$$
\begin{aligned}
J_{1} & \leq C \sum_{|\alpha|=m}\left\|D^{\alpha} b\right\|_{\operatorname{Lip}_{\beta}(w)} w(\tilde{Q})^{\beta / n} w(\tilde{x})|Q|^{-1 / s}\left(\int_{R^{n}}\left|f_{1}(x)\right|^{s} d x\right)^{1 / s} \\
& \leq C \sum_{|\alpha|=m}\left\|D^{\alpha} b\right\|_{L_{p_{\beta}(w)} w(\tilde{Q})^{\beta / n} w(\tilde{x})|Q|^{-1 / s}}
\end{aligned}
$$


Fens Journal of Inequalities and Applications 2014, 2014:188

Page 13 of 17

http://www.journalofinequalitiesandapplications.com/content/2014/1/188

$$
\begin{aligned}
& \times\left(\int_{\tilde{Q}}|f(x)|^{r} w(x) d x\right)^{1 / r}\left(\int_{\tilde{Q}} w(x)^{-s /(r-s)} d x\right)^{(r-s) / r s} \\
\leq & C \sum_{|\alpha|=m}\left\|D^{\alpha} b\right\|_{L i p_{\beta}(w)} w(\tilde{x})|\tilde{Q}|^{-1 / s} w(\tilde{Q})^{1 / r}\left(\frac{1}{w(\tilde{Q})^{1-r \beta / n}} \int_{\tilde{Q}}|f(x)|^{r} w(x) d x\right)^{1 / r} \\
& \times\left(\frac{1}{|\tilde{Q}|} \int_{\tilde{Q}} w(x)^{-s /(r-s)} d x\right)^{(r-s) / r s}\left(\frac{1}{|\tilde{Q}|} \int_{\tilde{Q}} w(x) d x\right)^{1 / r}|\tilde{Q}|^{1 / s} w(\tilde{Q})^{-1 / r} \\
\leq & C \sum_{|\alpha|=m}\left\|D^{\alpha} b\right\|_{L i p_{\beta}(w)} w(\tilde{x}) M_{\beta, r, w}(f)(\tilde{x}), \\
J_{2} \leq & C \sum_{|\alpha|=m} \frac{1}{|Q|} \int_{\tilde{Q}}\left|D^{\alpha} b(x)-\left(D^{\alpha} b\right)_{\tilde{Q}}\right| w(x)^{-1 / r}|f(x)| w(x)^{1 / r} d x \\
\leq & C \sum_{|\alpha|=m} \frac{1}{|Q|}\left(\int_{\tilde{Q}}\left|\left(D^{\alpha} b(x)-\left(D^{\alpha} b\right)_{\tilde{Q}}\right)\right|^{r^{\prime}} w(x)^{1-r^{\prime}} d x\right)^{1 / r^{\prime}}\left(\int_{\tilde{Q}}|f(x)|^{r} w(x) d x\right)^{1 / r} \\
\leq & C \sum_{|\alpha|=m} \frac{1}{|Q|}\left\|D^{\alpha} b\right\|_{L i p_{\beta}(w)} w(\tilde{Q})^{\beta / n+1 / r^{\prime}} w(\tilde{Q})^{1 / r-\beta / n}\left(\frac{1}{w(\tilde{Q})^{1-r \beta / n}} \int_{\tilde{Q}}|f(x)|^{r} w(x) d x\right)^{1 / r} \\
\leq & C \sum_{|\alpha|=m}\left\|D^{\alpha} b\right\|_{L i p_{\beta}(w)} \frac{w(\tilde{Q})}{|\tilde{Q}|} M_{\beta, r, w}(f)(\tilde{x}) \\
\leq & C \sum_{|\alpha|=m}\left\|D^{\alpha} b\right\|_{L i p_{\beta}(w)} w(\tilde{x}) M_{\beta, r, w}(f)(\tilde{x}) .
\end{aligned}
$$

For $J_{3}$, we have

$$
\begin{aligned}
& \left|R_{m}(\tilde{b} ; x, y)-R_{m}\left(\tilde{b} ; x_{0}, y\right)\right| \\
& \quad \leq C \sum_{|\gamma|<m} \sum_{|\alpha|=m}\left|x-x_{0}\right|^{m-|\gamma|}|x-y|^{|\gamma|}\left\|D^{\alpha} b\right\|_{L_{i p_{\beta}(w)} w(\tilde{x}) w\left(2^{k} \tilde{Q}\right)}
\end{aligned}
$$

and

$$
\left|R_{m}(\tilde{b} ; x, y)\right| \leq C \sum_{|\alpha|=m}\left|x-x_{0}\right|^{m}\left\|D^{\alpha} b\right\|_{L_{i_{\beta}}(w)} w(\tilde{x}) w\left(2^{k} \tilde{Q}\right) .
$$

Thus, for $1<p<\infty$ with $1 / p+1 / q+1 / r=1$ and $r<p_{1}^{\prime}<\infty, 1<s_{1}, s_{2}<\infty$ with $1 / p_{1}+1 / q+$ $1 / r+1 / s_{1}=1$ and $1 / q+1 / r+1 / s_{2}=1$, we obtain

$$
\begin{aligned}
& \left|T^{\tilde{b}}\left(f_{2}\right)(x)-T^{\tilde{b}}\left(f_{2}\right)\left(x_{0}\right)\right| \\
& \quad \leq \int_{R^{n}}\left|R_{m}(\tilde{b} ; x, y)-R_{m}\left(\tilde{b} ; x_{0}, y\right)\right| \frac{|K(x, y)|}{|x-y|^{m}}\left|f_{2}(y)\right| d y \\
& \quad+\int_{R^{n}}\left|\frac{K(x, y)}{|x-y|^{m}}-\frac{K\left(x_{0}, y\right)}{\left|x_{0}-y\right|^{m}}\right|\left|R_{m}\left(\tilde{b} ; x_{0}, y\right)\right|\left|f_{2}(y)\right| d y \\
& \quad+\sum_{|\alpha|=m} \frac{1}{\alpha !} \int_{R^{n}}\left|\frac{K(x, y)(x-y)^{\alpha}}{|x-y|^{m}}-\frac{K\left(x_{0}, y\right)\left(x_{0}-y\right)^{\alpha}}{\left|x_{0}-y\right|^{m}}\right|\left|D^{\alpha} \tilde{b}(y)\right|\left|f_{2}(y)\right| d y \\
& \leq C \sum_{|\alpha|=m}\left\|D^{\alpha} b\right\|_{L_{i p}(w)} w(\tilde{x}) \sum_{k=0}^{\infty} w\left(2^{k+1} \tilde{Q}\right)^{\beta / n} \int_{2^{k+1} \tilde{Q} \backslash 2^{k} \tilde{Q}} \frac{\left|x-x_{0}\right|}{\left|x_{0}-y\right|^{n+1}}|f(y)| d y
\end{aligned}
$$


Fen Journal of Inequalities and Applications 2014, 2014:188

Page 14 of 17

http://www.journalofinequalitiesandapplications.com/content/2014/1/188

$$
\begin{aligned}
& +C \sum_{|\alpha|=m}\left\|D^{\alpha} b\right\|_{L i i_{\beta}(w)} w(\tilde{x}) \sum_{k=0}^{\infty} w\left(2^{k+1} \tilde{Q}\right)^{\beta / n}\left(\int_{2^{k+1} \tilde{Q} 2^{k} \tilde{Q}}\left|K(x, y)-K\left(x_{0}, y\right)\right|^{q} d y\right)^{1 / q} \\
& \times\left(\int_{2^{k+1} \tilde{Q} \backslash 2^{k} \tilde{Q}}|f(y)|^{r} w(y) d y\right)^{1 / r}\left(\int_{2^{k+1} \tilde{Q} \backslash 2^{k} \tilde{Q}} w(y)^{-p / r} d y\right)^{1 / p}
\end{aligned}
$$

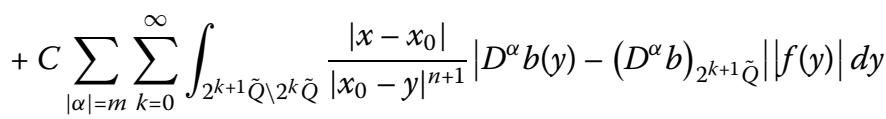

$$
\begin{aligned}
& +C \sum_{|\alpha|=m} \sum_{k=0}^{\infty} \int_{2^{k+1} \tilde{Q} \backslash 2^{k} \tilde{Q}} \frac{\left|x-x_{0}\right|}{\left|x_{0}-y\right|^{n+1}}\left|\left(D^{\alpha} b\right)_{2^{k+1} \tilde{Q}}-\left(D^{\alpha} b\right)_{\tilde{Q}}\right| f(y) \mid d y \\
& +C \sum_{|\alpha|=m} \sum_{k=0}^{\infty}\left(\int_{2^{k+1} \tilde{Q} \backslash 2^{k} \tilde{Q}}\left|K(x, y)-K\left(x_{0}, y\right)\right|^{q} d y\right)^{1 / q}\left(\int_{2^{k+1} \tilde{Q}}|f(y)|^{r} w(y) d y\right)^{1 / r} \\
& \times\left(\int_{2^{k+1} \tilde{Q}}\left|D^{\alpha} b(y)-\left(D^{\alpha} b\right)_{2^{k+1} \tilde{Q}}\right|^{p_{1}} w(y)^{1-p_{1}} d y\right)^{1 / p_{1}}\left(\int_{2^{k+1} \tilde{Q}} w(y)^{-\left(1 / r-1 / p_{1}^{\prime}\right) s_{1}} d y\right)^{1 / s_{1}} \\
& +C \sum_{|\alpha|=m} \sum_{k=0}^{\infty}\left(\int_{2^{k+1} \tilde{Q} 2^{k} \tilde{Q}}\left|K(x, y)-K\left(x_{0}, y\right)\right|^{q} d y\right)^{1 / q}\left(\int_{2^{k+1} \tilde{Q}}|f(y)|^{r} w(y) d y\right)^{1 / r} \\
& \times\left|\left(D^{\alpha} b\right)_{2^{k+1} \tilde{Q}}-\left(D^{\alpha} b\right)_{\tilde{Q}}\right|\left(\int_{2^{k+1} \tilde{Q}} w(y)^{-s_{2} / r} d y\right)^{1 / s_{2}}
\end{aligned}
$$

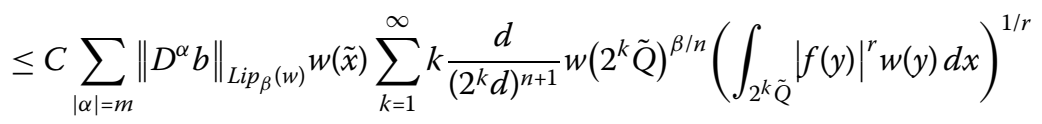

$$
\begin{aligned}
& \times\left(\frac{1}{\left|2^{k} \tilde{Q}\right|} \int_{2^{k} \tilde{Q}} w(y)^{-1 /(r-1)} d y\right)^{(r-1) / r}\left(\frac{1}{\left|2^{k} \tilde{Q}\right|} \int_{2^{k} \tilde{Q}} w(y) d y\right)^{1 / r}\left|2^{k} \tilde{Q}\right| w\left(2^{k} \tilde{Q}\right)^{-1 / r} \\
& +C \sum_{|\alpha|=m}\left\|D^{\alpha} b\right\|_{L_{i p_{\beta}(w)}} w(\tilde{x}) \sum_{k=1}^{\infty} C_{k}\left|2^{k} Q\right|^{-1 / q^{\prime}} w\left(2^{k} \tilde{Q}\right)^{\beta / n}\left(\int_{2^{k} \tilde{Q}}|f(y)|^{r} w(y) d x\right)^{1 / r} \\
& \times\left(\frac{1}{\left|2^{k} \tilde{Q}\right|} \int_{2^{k} \tilde{Q}} w(y)^{-p / r} d y\right)^{1 / p}\left(\frac{1}{\left|2^{k} \tilde{Q}\right|} \int_{2^{k} \tilde{Q}} w(y) d y\right)^{1 / r}\left|2^{k} \tilde{Q}\right|^{1 / p+1 / r} w\left(2^{k} \tilde{Q}\right)^{-1 / r} \\
& +C \sum_{|\alpha|=m} \sum_{k=1}^{\infty} \frac{d}{\left(2^{k} d\right)^{n+1}}\left(\int_{2^{k} \tilde{Q}}\left|D^{\alpha} b(y)-\left(D^{\alpha} b\right)_{2^{k} \tilde{Q}}\right|^{r^{\prime}} w(y)^{1-r^{\prime}} d y\right)^{1 / r^{\prime}} \\
& \times\left(\int_{2^{k} \tilde{Q}}|f(y)|^{r} w(y) d y\right)^{1 / r} \\
& +C \sum_{|\alpha|=m}\left\|D^{\alpha} b\right\|_{\operatorname{Lip}_{\beta}(w)} w(\tilde{x}) \sum_{k=1}^{\infty} C_{k}\left|2^{k} Q\right|^{-1 / q^{\prime}} w\left(2^{k} \tilde{Q}\right)^{\beta / n}\left(\int_{2^{k} \tilde{Q}}|f(y)|^{r} w(y) d x\right)^{1 / r} \\
& \times\left(\frac{1}{\left|2^{k} \tilde{Q}\right|} \int_{2^{k} \tilde{Q}} w(y)^{-\left(1 / r-1 / p_{1}^{\prime}\right) s_{1}} d y\right)^{1 / s_{1}}\left(\frac{1}{\left|2^{k} \tilde{Q}\right|} \int_{2^{k} \tilde{Q}} w(y) d y\right)^{1 / r-1 / p_{1}^{\prime}} \\
& \times\left|2^{k} \tilde{Q}\right|^{1 / s_{1}+1 / r-1 / p_{1}^{\prime}} w\left(2^{k} \tilde{Q}\right)^{-1 / r+1 / p_{1}^{\prime}} \\
& +C \sum_{|\alpha|=m}\left\|D^{\alpha} b\right\|_{L_{p_{\beta}(w)} w(\tilde{x})} \sum_{k=1}^{\infty} k C_{k}\left|2^{k} Q\right|^{-1 / q^{\prime}} w\left(2^{k} \tilde{Q}\right)^{\beta / n}\left(\int_{2^{k} \tilde{Q}}|f(y)|^{r} w(y) d y\right)^{1 / r} \\
& \times\left(\frac{1}{\left|2^{k} \tilde{Q}\right|} \int_{2^{k} \tilde{Q}} w(y) d y\right)^{1 / r}\left(\frac{1}{\left|2^{k} \tilde{Q}\right|} \int_{2^{k} \tilde{Q}} w(y)^{-s_{2} / r} d y\right)^{1 / s_{2}}\left|2^{k} \tilde{Q}\right|^{1 / s_{2}+1 / r} w\left(2^{k} \tilde{Q}\right)^{-1 / r}
\end{aligned}
$$




$$
\begin{aligned}
& \leq C \sum_{|\alpha|=m}\left\|D^{\alpha} b\right\|_{L_{i p_{\beta}(w)}} w(\tilde{x}) \sum_{k=1}^{\infty} k\left(C_{k}+2^{-k}\right) \\
& \quad \times\left(\frac{1}{w\left(2^{k} \tilde{Q}\right)^{1-r \beta / n}} \int_{2^{k} \tilde{Q}}|f(y)|^{r} w(y) d x\right)^{1 / r} \\
& \quad+C \sum_{|\alpha|=m}\left\|D^{\alpha} b\right\|_{{L i p_{\beta}}(w)} \sum_{k=1}^{\infty} k\left(C_{k}+2^{-k}\right) \frac{w\left(2^{k} \tilde{Q}\right)}{\left|2^{k} \tilde{Q}\right|} \\
& \quad \times\left(\frac{1}{w\left(2^{k} \tilde{Q}\right)^{1-r \beta / n}} \int_{2^{k} \tilde{Q}}|f(y)|^{r} w(y) d x\right)^{1 / r} \\
& \leq C \sum_{|\alpha|=m}\left\|D^{\alpha} b\right\|_{L_{i p_{\beta}(w)}} w(\tilde{x}) M_{\beta, r, w}(f)(\tilde{x}) .
\end{aligned}
$$

This completes the proof of Theorem 2 .

Proof of Theorem 3 Choose $1<r<u$ in Theorem 1 and notice $w^{1-u} \in A_{1}$, then we have, by Lemmas 3 and 4 ,

$$
\begin{aligned}
\left\|T^{b}(f)\right\|_{L^{u}\left(w^{1-u}\right)} & \leq\left\|M_{\eta}\left(T^{b}(f)\right)\right\|_{L^{u}\left(w^{1-u}\right)} \leq C\left\|M_{\eta}^{\#}\left(T^{b}(f)\right)\right\|_{L^{u}\left(w^{1-u}\right)} \\
& \leq C \sum_{|\alpha|=m}\left\|D^{\alpha} b\right\|_{B M O(w)}\left\|w M_{r, w}(f)\right\|_{L^{u}\left(w^{1-u}\right)} \\
& =C \sum_{|\alpha|=m}\left\|D^{\alpha} b\right\|_{B M O(w)}\left\|M_{r, w}(f)\right\|_{L^{u}(w)} \\
& \leq C \sum_{|\alpha|=m}\left\|D^{\alpha} b\right\|_{B M O(w)}\|f\|_{L^{u}(w)} .
\end{aligned}
$$

This completes the proof of Theorem 3.

Proof of Theorem 4 Choose $1<r<u$ in Theorem 1 and notice $w^{1-u} \in A_{1}$, then we have, by Lemmas 5 and 6 ,

$$
\begin{aligned}
\left\|T^{b}(f)\right\|_{L^{u, \varphi}\left(w^{1-u}\right)} & \leq\left\|M_{\eta}\left(T^{b}(f)\right)\right\|_{L^{u, \varphi}\left(w^{1-u}\right)} \leq C\left\|M_{\eta}^{\#}\left(T^{b}(f)\right)\right\|_{L^{u, \varphi}\left(w^{1-u}\right)} \\
& \leq C \sum_{|\alpha|=m}\left\|D^{\alpha} b\right\|_{B M O(w)}\left\|w M_{r, w}(f)\right\|_{L^{u, \varphi}\left(w^{1-u}\right)} \\
& =C \sum_{|\alpha|=m}\left\|D^{\alpha} b\right\|_{B M O(w)}\left\|M_{r, w}(f)\right\|_{L^{u, \varphi}(w)} \\
& \leq C \sum_{|\alpha|=m}\left\|D^{\alpha} b\right\|_{B M O(w)}\|f\|_{L^{u, \varphi}(w) .}
\end{aligned}
$$

This completes the proof of Theorem 4 .

Proof of Theorem 5 Choose $1<r<u$ in Theorem 2 and notice $w^{1-v} \in A_{1}$, then we have, by Lemmas 3 and 4 ,

$$
\begin{aligned}
\left\|T^{b}(f)\right\|_{L^{\nu}\left(w^{1-\nu}\right)} & \leq\left\|M_{\eta}\left(T^{b}(f)\right)\right\|_{L^{v}\left(w^{1-v}\right)} \leq C\left\|M_{\eta}^{\#}\left(T^{b}(f)\right)\right\|_{L^{v}\left(w^{1-\nu}\right)} \\
& \leq C \sum_{|\alpha|=m}\left\|D^{\alpha} b\right\|_{L i p_{\beta}(w)}\left\|w M_{\beta, r, w}(f)\right\|_{L^{v}\left(w^{1-\nu}\right)}
\end{aligned}
$$




$$
\begin{aligned}
& =C \sum_{|\alpha|=m}\left\|D^{\alpha} b\right\|_{L_{L p_{\beta}(w)}\left\|M_{\beta, r, w}(f)\right\|_{L^{v}(w)}} \\
& \leq C \sum_{|\alpha|=m}\left\|D^{\alpha} b\right\|_{L i p_{\beta}(w)}\|f\|_{L^{u}(w) .} .
\end{aligned}
$$

This completes the proof of Theorem 5 .

Proof of Theorem 6 Choose $1<r<u$ in Theorem 2 and notice $w^{1-v} \in A_{1}$, then we have, by Lemmas 5 and 6 ,

$$
\begin{aligned}
\left\|T^{b}(f)\right\|_{L^{v, \varphi}\left(w^{1-v}\right)} & \leq\left\|M_{\eta}\left(T^{b}(f)\right)\right\|_{L^{v, \varphi}\left(w^{1-v}\right)} \leq C\left\|M_{\eta}^{\#}\left(T^{b}(f)\right)\right\|_{L^{v, \varphi}\left(w^{1-v}\right)} \\
& \leq C \sum_{|\alpha|=m}\left\|D^{\alpha} b\right\|_{L i p_{\beta}(w)}\left\|w M_{\beta, r, w}(f)\right\|_{L^{v, \varphi}\left(w^{1-v}\right)} \\
& =C \sum_{|\alpha|=m}\left\|D^{\alpha} b\right\|_{L i p_{\beta}(w)}\left\|M_{\beta, r, w}(f)\right\|_{L^{v, \varphi}(w)} \\
& \leq C \sum_{|\alpha|=m}\left\|D^{\alpha} b\right\|_{L i p_{\beta}(w)}\|f\|_{L^{u, \varphi}(w) .}
\end{aligned}
$$

This completes the proof of Theorem 6 .

\section{Competing interests}

The author declares that they have no competing interests.

\section{Received: 24 December 2013 Accepted: 25 April 2014 Published: 13 May 2014}

\section{References}

1. Garcia-Cuerva, J, Rubio de Francia, JL: Weighted Norm Inequalities and Related Topics. North-Holland Mathematics Studies, vol. 16. North-Holland, Amsterdam (1985)

2. Stein, EM: Harmonic Analysis: Real Variable Methods, Orthogonality and Oscillatory Integrals. Princeton University Press, Princeton (1993)

3. Torchinsky, A: Real Variable Methods in Harmonic Analysis. Pure and Applied Math., vol. 123. Academic Press, New York (1986)

4. Coifman, RR, Rochberg, R, Weiss, G: Factorization theorems for Hardy spaces in several variables. Ann. Math. 103 611-635 (1976)

5. Pérez, C: Endpoint estimate for commutators of singular integral operators. J. Funct. Anal. 128, 163-185 (1995)

6. Pérez, C, Trujillo-Gonzalez, R: Sharp weighted estimates for multilinear commutators. J. Lond. Math. Soc. 65, 672-692 (2002)

7. Chanillo, S: A note on commutators. Indiana Univ. Math. J. 31, 7-16 (1982)

8. Lin, Y: Sharp maximal function estimates for Calderón-Zygmund type operators and commutators. Acta Math. Sci., Ser. A 31, 206-215 (2011)

9. Paluszynski, M: Characterization of the Besov spaces via the commutator operator of Coifman, Rochberg and Weiss Indiana Univ. Math. J. 44, 1-17 (1995)

10. Bloom, S: A commutator theorem and weighted BMO. Trans. Am. Math. Soc. 292, 103-122 (1985)

11. $\mathrm{Hu}, \mathrm{B}, \mathrm{Gu}, \mathrm{J}$ : Necessary and sufficient conditions for boundedness of some commutators with weighted Lipschitz spaces. J. Math. Anal. Appl. 340, 598-605 (2008)

12. Chang, DC, Li, JF, Xiao, J: Weighted scale estimates for Calderón-Zygmund type operators. Contemp. Math. 446, 61-70 (2007)

13. Liu, LZ: Sharp maximal function estimates and boundedness for commutators associated with general integral operator. Filomat 25(4), 137-151 (2011)

14. Garcia-Cuerva, J: Weighted $H^{p}$ spaces. Diss. Math. 162, 1-63 (1979)

15. Peetre, J: On convolution operators leaving $L^{p, \lambda}$-spaces invariant. Ann. Mat. Pura Appl. 72, 295-304 (1966)

16. Peetre, J: On the theory of $L^{p, \lambda}$-spaces. J. Funct. Anal. 4, 71-87 (1969)

17. Di Fazio, G, Ragusa, MA: Commutators and Morrey spaces. Boll. Unione Mat. Ital., A 5, 323-332 (1991)

18. Di Fazio, G, Ragusa, MA: Interior estimates in Morrey spaces for strong solutions to nondivergence form equations with discontinuous coefficients. J. Funct. Anal. 112, 241-256 (1993)

19. Liu, LZ: Interior estimates in Morrey spaces for solutions of elliptic equations and weighted boundedness for commutators of singular integral operators. Acta Math. Sci., Ser. B 25, 89-94 (2005)

20. Mizuhara, T: Boundedness of some classical operators on generalized Morrey spaces. In: Harmonic Analysis: Proceedings of a Conference Held in Sendai, Japan, pp. 183-189 (1990) 
21. Cohen, J, Gosselin, J: On multilinear singular integral operators on $R^{n}$. Stud. Math. 72, 199-223 (1982)

22. Cohen, J, Gosselin, J: A BMO estimate for multilinear singular integral operators. III. J. Math. 30, 445-465 (1986)

23. Ding, Y, Lu, SZ: Weighted boundedness for a class rough multilinear operators. Acta Math. Sin. 17, 517-526 (2001)

10.1186/1029-242X-2014-188

Cite this article as: Feng: Weighted boundedness of a multilinear operator associated to a singular integral operator with general kernels. Journal of Inequalities and Applications 2014, 2014:188

Submit your manuscript to a SpringerOpen ${ }^{\circ}$ journal and benefit from:

- Convenient online submission

- Rigorous peer review

- Immediate publication on acceptance

- Open access: articles freely available online

- High visibility within the field

- Retaining the copyright to your article

Submit your next manuscript at $>$ springeropen.com 